\title{
Rhinolith
}

\section{A brief review of the literature and a case report}

\author{
P. T. MARFATIA* \\ M.S., D.O.R.L., D.L.O.
}

The Royal Infirmary, Preston, Lancashire

The SUBJECT of nasal calculi has intrigued men since the very beginning of western medical literature. Polson (1943) reports that as early as 1502 Mathias di Gardi mentions that a colleague had seen a rhinolith as big as a pinecone expelled by a patient. The first welldocumented case of rhinolithiasis was reported by Bartholin in 1654 . Since then, over 400 cases have been described. Axmann was the first person to analyse a rhinolith, in 1829 . It has been shown that they occur more commonly in females, and in the lower half of the nasal cavity.

Rhinoliths are calcareous bodies occasionally found in the nose and, very rarely, in the maxillary antrum. They are almost always single and unilateral, are more or less spherical, and are grey or brownish-black in colour. They consist chiefly of calcium phosphate and carbonate, which originates mainly from the inflammatory exudates but also from the nasal mucus and tears.

On section, there is evidence that the salts have been deposited around a nucleus which may be endogenous or exogenous in origin. An endogenous mucleus could be blood, mucus, sequestra or nasal crusts. An exogenous nucleus could be any small foreign body such as a bead, button or fruit-stone, most commonly a cherry stone. Van Alyea (1952) states that 'as far as it is known, this, the endogenous nature of the growth is purely theoretical and no evidence that these calculi may form around a matrix of dried pus or other endogenous material, has been presented'.

The symptoms of rhinoliths are those of a nasal foreign body, commonly, unilateral purulent nasal discharge, obstruction and ozena.

The diagnosis of these stones, both large and small, is usually made by inspection with the aid of a probe. In most cases, the diagnosis,

*Present address : 26A Walkeshwar Road, Bombay 6, India. and the evaluation of the destructive effects on the nasal interior, can be made by radiology. Rhinoliths may have to be differentiated from a calcified polyp, an osteoma, osteomyelitis and a carcinoma (Hunt, Cotton \& Joseph, 1966).

The treatment is removal. If it is large, it should be crushed and the fragments removed as in the case described below. With the largest stones, radical surgery may be neeessary, as in the cases described by Abu-Jaudeh (1951) and Myerson (1928).

\section{Case report}

A female, M.S., aged 54 years, attended the E.N.T. Outpatients' Department on 8 June 1967, complaining of nasal obstruction on the right side for about 3 months. There was a history of yellow thick discharge from the nose.

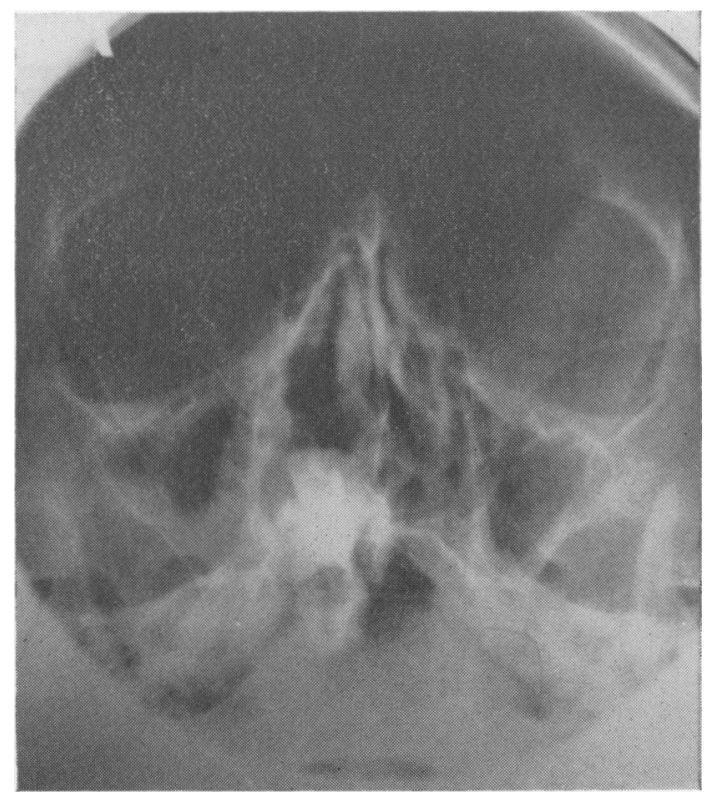

Fig. 1. X-ray view of the rhinolith in the floor of the nose. 
There was no history of a foreign body in the nose at any time before.

Anterior rhinoscopy showed a rough dark stony mass covered with greenish discharge. Probing gave a metallic sound. Postnasal examination showed a greenish discharge. A clinical diagnosis of a rhinolith was made. Radiology showed the rhinolith's position (Fig. 1) in the floor of the right nostril with mucosal thickening of the right maxillary antrum. The nasal swab showed a growth of $B$. proteus, sensitive

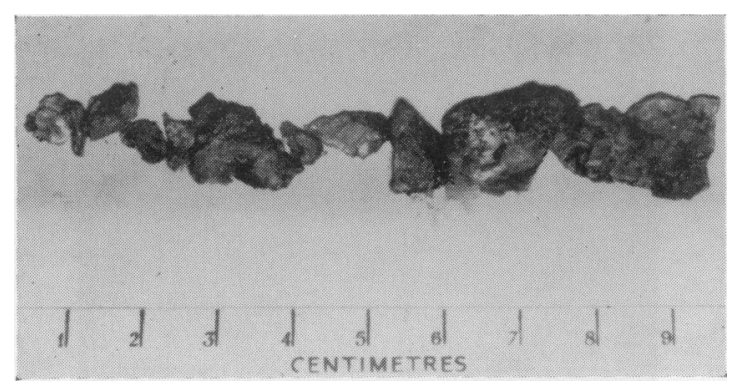

FIG. 2. Photograph of the pieces after removal. to streptomycin, chloramphenicol and ampicillin.

This stone was removed in pieces under general anaesthesia (Fig. 2). Small pieces were fished out from the postnasal space. The nasal cavity was washed out with normal saline.

The patient was given antibiotics for 7 days. She had an uneventful recovery after the operation.

The stone weighed $4.4 \mathrm{~g}$; chemical analysis showed that it consisted of calcium and magnesium, oxalates and urates.

\section{Acknowledgments}

I acknowledge with pleasure and gratitude the help given by Mr H. Wickham, Mr J. A. Kersley and Mr A. Robinson.

\section{References}

AbU-Jaudeh, C.N. (1951) A giant rhinolith. Laryngoscope, 61, 27.

HuNT, W.L., CotTon, B.P. \& JoSEPH, D.J. (1966) Rhinoliths. Arch. Otolaryng. 83, 256.

MYERSON, M.C. (1928) Rhinolith requiring external nasal operation for its removal. Laryngoscope, 38, 393.

Polson, C.J. (1943) On rhinoliths. J. Laryng. 58, 79.

VAN AlYeA, O.E. (1952) Rhinolith: a report of two cases. Ann. Oto-laryng. (Paris), 61, 490.

\section{Unusual presentation of intralobar pulmonary sequestration}

\author{
Brig. H. B. LAL \\ F.R.C.P.(E), D.C.H. \\ Consultant in Medicine
P. N. Dhawad
M.B.B.S.
Registrar

\author{
A. L. BAHL \\ M.D. \\ Assistant Surgeon
}
A. K. Sharma
M.S.
Registrar

\author{
S. MUKHERJEE \\ F.R.C.S.(Eng.), F.R.C.S.(Edin.), \\ F.A.C.S. \\ Associate Professor of Surgery \\ G. S. SARIN
R.C.P.(Canada)
Senior Radiologist
}

Departments of Medicine, Surgery and Radiology, Willingdon Hospital, New Delhi, India

BRONCHOPULMONARY sequestration was first described adequately by Pryce (1946), only three such case reports having been published till then. A total of about 150 surgically treated cases had been reported up till 1966 (Rubin et al., 1966). The intralobar variety consists of a bronchopulmonary mass or cyst enveloped in the same pleura as the normal lung and has an abnormal blood supply by an aberrant vessel originating from the aorta in the vicinity of the diaphragm. This ectopic pulmonary mass does not communicate with the normal bronchial tree. In the extralobar type, the lung mass is completely separate from the lung and has no connection with the arteries and veins of the pulmonary circulation (James \& Cuthbert, 1967)

Pulmonary sequestration is often detected on operation or autopsy although pre-operative diagnosis has been made by aortography (Turk \& Lindskog, 1961 ; Sutton \& Samuel, 1963 ; Ranniger \& Valvassori, 1964 ; Rubin et al., 1966). The clinical diagnosis in most of these patients is empyema, lung abscess, bronchiectasis, or recurrent pneumonia (Borrie, Lichter \& Rodda, 1963). The case described below is of interest because of its unusual mode of presentation.

\section{Case report}

V.K., a 16-year-old male, was admitted on 7 December 1966 with a history of fever of 